\title{
Application of polarization ellipse technique for analysis of ULF magnetic fields from two distant stations in Koyna-Warna seismoactive region, West India
}

\author{
F. Dudkin ${ }^{1}$, Gautam Rawat ${ }^{2}$, B. R. Arora ${ }^{2}$, V. Korepanov ${ }^{1}$, O. Leontyeva ${ }^{1,{ }^{*}}$, and A. K. Sharma ${ }^{3}$ \\ ${ }^{1}$ Lviv Centre of Institute of Space Research, Lviv, 79000, Ukraine \\ ${ }^{2}$ Wadia Institute of Himalayan Geology, Dehradun, 248 001, India \\ ${ }^{3}$ Department of Physics, Shivaji University, Kolhapur, India \\ * Invited contribution by $O$. Leontyeva, recipient of the EGU \\ Young Scientists Outstanding Poster Paper Award 2008.
}

Received: 26 November 2009 - Revised: 15 June 2010 - Accepted: 16 June 2010 - Published: 12 July 2010

\begin{abstract}
A new approach is developed to find the source azimuth of the ultra low frequency (ULF) electromagnetic (EM) signals believed to be emanating from well defined seismic zone. The method is test applied on magnetic data procured from the seismoactive region of Koyna-Warna, known for prolonged reservoir triggered seismicity. Extremely low-noise, high-sensitivity LEMI-30 search coil magnetometers were used to measure simultaneously the vector magnetic field in the frequency range $0.001-32 \mathrm{~Hz}$ at two stations, the one located within and another $\sim 100 \mathrm{~km}$ away from the seismic active zone. During the observation campaign extending from 15 March to 30 June 2006 two earthquakes (EQs) of magnitude $\left(\mathrm{M}_{L}>4\right)$ occurred, which are searched for the presence of precursory EM signals.

Comparison of polarization ellipses (PE) parameters formed by the magnetic field components at the measurement stations, in select frequency bands, allows discrimination of seismo-EM signals from the natural background ULF signals of magnetospheric/ionospheric origin. The magnetic field components corresponding to spectral bands dominated by seismo-EM fields define the PE plane which at any instant contains the source of the EM fields. Intersection lines of such defined PE planes for distant observation stations clutter in to the source region. Approximating the magnetic-dipole configuration for the source, the magnetic field components along the intersection lines suggest that azimuth of the EM source align in the NNW-SSE direction. This direction well
\end{abstract}

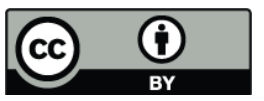

Correspondence to: Gautam Rawat (rawatg@wihg.res.in) coincides with the orientation of nodal plane of normal fault plane mechanism for the two largest EQs recorded during the campaign. More significantly the correspondence of this direction with the tectonic controlled trend in local seismicity, it has been surmised that high pressure fluid flow along the fault that facilitate EQs in the region may also be the source mechanism for EM fields by electrokinetic effect.

\section{Introduction}

Short-term earthquake (EQ) prediction, despite intensive efforts in last half a century, still remains unattainable though numbers of promising leads and directions are indicated (see Uyeda et al., 2009, for recent review on the subject). The anomalous electromagnetic (EM) emission in ultra low frequency (ULF) band $(0.001-10 \mathrm{~Hz})$, believed to be emanating from within the focal zones, have emerged as potential precursor candidates for short-term EQ prediction (Hayakawa et al., 1996, 2000, 2004, 2007; Molchanov and Hayakawa, 1995; Molchanov et al., 1992, 2004). This observational conviction is further reinforced from the suggestions that mechanical deformations or microfracturing in the impending focal zones may give rise to pre- and/or co-seismic EM emission in ULF band due to one or more of the following factors: (1) inductive effect resulting from the movement of conductive medium in the Earth's permanent magnetic field [Surkov, 1999; Surkov et al., 2003]; (2) displacements of boundaries between high and low conductive crustal blocks (Dudkin et al., 2003); (3) electrokinetic effect (Mizutani et al., 1976; Fitterman, 1979; Fedorov et al., 2001); (4) piezoelectric or

Published by Copernicus Publications on behalf of the European Geosciences Union. 
piezomagnetic effects (Martin et al., 1978; Ogawa et al., 1985; Johnston et al., 1994; Ogawa and Utada, 2000) and (5) microfracture electrification (Molchanov and Hayakawa, 1995) (all references are given as example). The underground ULF EM field attenuates only little in crustal material and hence on theoretical consideration associated magnetic field can be detected to a large distances up to $100-150 \mathrm{~km}$ (Hayakawa et al., 2007).

The practical detections and applications of precursory EM signals in real time EQ prediction continue to be challenging due to several problems; (i) intensity of anticipated seismo-EM signals in ULF band is very low (notable exception being the highly enhanced signals recorded in association with M7.1 Loma Prieta earthquake (Fraser-Smith et al., 1990; Bleier et al., 2009), although question has been raised that this anomalously large signal may not be due the proximity of the magnetometer to the epicenter but artifact of the sensor-system malfunction (Thomas et al., 2009)), (ii) discrimination of weak seismo-EM signals from the background natural EM fields of ionospheric and magnetospheric origin and (iii) finally the limitation in the localization of precursor source or, at least, determination of azimuth direction to the source zone. Very often later problems are aggravated by short time (less than $5 \mathrm{~min}$ ) of precursor existence (Bleier et al., 2009). With the availability of very sensitive induction type 3-component magnetometers with high suppression of man-made interference, the recording of high quality magnetic data in ULF bands has greatly improved (Hayakawa et al., 2007). For the second problem, polarization analysis incorporating the ratio $S_{Z} / S_{H}\left(S_{Z}\right.$ and $S_{H}$ are the spectral intensities of vertical and horizontal magnetic field components) is found effective, at least partially, in distinguishing seismo-EM signals from geomagnetic field fluctuations (Hayakawa et al., 1996). The formulations of principal component analysis and fractal approach have been used with some success in isolating components of extra-terrestrial and seismotectonic origin in magnetic field records (see, for example, Hayakawa et al., 1999, 2007; Serita et al., 2005; Ida and Hayakawa, 2006). Towards the identification of underground ULF source or its direction, formulation based on the time lag or phase difference between pair or more observation points has been advanced, referred to as gradiometric method (Kopytenko et al., 2001, 2006; Ismaguilov et al., 2003). The technique may lack reliability as the ULF electromagnetic waves propagating through the conductive layers undergo strong dissipation and dispersions, making the identification of front of ULF signal ambiguous (Surkov et al., 2004). Working independently, Surkov et al. (2004) advocated use of amplitude difference in synchronous observation at two or multiple recording sites. The method, in principle, hold promise to estimate both the location and direction of the ULF source provided the spatial scale of natural noise variation should be much greater than both characteristic length of the ULF signal itself and distance between magnetometers. However, space derivative of magnetic field pertur- bations tend to be very unstable at low signal-to-noise $(\mathrm{S} / \mathrm{N})$ ratio and gives a big error in the estimation of source direction (see remarks about S/N ratio in Dudkin et al., 2003). Very promising in the direction-finding problem for seismoEM precursors is an application of the polarization ellipse (PE) technique, where the PE major axis behavior is investigated (goniometric method) (Du et al., 2002; Schekotov et al., 2007, 2008). This technique allows determination of trends in azimuth angle of anomalous ULF signal and possibly area of EQ epicentre. Taking into account that ULF magnetic source is always in the PE plane (see Appendix) the new method of magnetic precursor source location from two observation points has been proposed by present authors (Dudkin et al., 2008). In the present paper expanding on the steps of this new direction-finding approach, we test apply the formulation on magnetic field data from pair of stations operated simultaneously in one of the seismoactive region of India.

The paper is structured as follow: Sect. 2 gives brief account of the seismic background in the study area. The noisesensitivity parameters of magnetometer deployed, data acquisition and initial data preparation steps and nature of the seismic activity recorded during the observational campaign are listed in Sect. 3. The outline of the PE method for the isolation of seismo-EM signals and estimation of the direction and possible location of seismo-EM source is described in Sect. 4. In Sect. 5, discussion of the results in reference to source validation and possible generating source mechanism form the central stage, followed by a short summary.

\section{Current and past seismic history of the study area and experiment description}

Koyna-Warna region in the southern part of Deccan Volcanic Province, Western India is a classical example of reservoir triggered seismicity - Fig. 1 (for review see Gupta, 2002, and references therein). The largest triggered earthquake of M 6.3 occurred here on 10 December 1967, and over the past four and a half decades area has remained intensively seismoactive (Gupta, 2005). During this period $19 \mathrm{EQs}$ of $\mathrm{M}_{L} \geq 5$ and about 170 EQs with $\mathrm{M}_{L} \geq 4$ have occurred, all confined to a well defined belt of roughly $20 \times 30 \mathrm{~km}^{2}$ with hypocentres $h \leq 12 \mathrm{~km}$ (Gupta et al., 2007). Such features make the area unique for studying peculiarities of magnetic field during EQ preparation process.

To develop and test the PE method for locating source region of EM fields produced during EQ preparation process, two stations to record magnetic variations in ULF bands were established; the first at Koyna, within the limits of focused seismic zone, and other was placed at distant location of $\sim 100 \mathrm{~km}$ in Shivaji University, Kolhapur (Fig. 1). Both sites were located in low-noise background and have minimal interference from man-made disturbances. 
Extremely low-noise $\left(S_{B, n}=0.2 \mathrm{pT} / \mathrm{Hz}^{0.5}\right.$ at $f=1 \mathrm{~Hz}$, where $S_{B, n}$ is spectral noise density) with high factor of industrial interference suppression (more than 1000) 3component LEMI-30 magnetometers, specially designed by Lviv Centre of Institute for Space Research, Ukraine (http: //www.isr.lviv.ua) for EQ EM monitoring, were deployed at both stations for synchronous recording. LEMI-30 magnetometers operate in frequency range $0.001-32 \mathrm{~Hz}$ and are ideally suited to record the most promising EQ magnetic precursors in ULF band, which are found dominant below $0.1 \mathrm{~Hz}$ (Hayakawa et al., 2004, 2007). Both stations recorded simultaneous data with 64 samples per second over the entire observational campaign period of 15 March-30 June 2006. During data processing a resampling procedure was applied with averaging each of 64 samples. Thus an upper boundary of signal spectra was decreased to $0.5 \mathrm{~Hz}$. For such data the dynamical Fourier spectra (DFS) for each $24 \mathrm{~h}$ of data recording were calculated. Then for each point of DFS the parameters of PE for each measuring site were calculated, which form the base to search EQ precursory magnetic analysis described in the next section.

In the post 1993 period, the seismic activity in KoynaWarna region is being monitored by a closely spaced network of seven modern 3-component seismometers established by National Geophysical Research Institute, Hyderabad (Gupta, 2002). The uniform azimuth coverage allows estimation of epicentres to an accuracy of $\pm 0.5 \mathrm{~km}$. During the period of present campaign, more than $700 \mathrm{EQs}$ with magnitude in the range of $\mathrm{M}_{L}=0.5-4.7$ were recorded, two of them with magnitude $\mathrm{M}_{L}>4,8 \mathrm{EQs}$ with $\mathrm{M}_{L}>3$ and as many as 172 with $\mathrm{M}_{L}>2$. The spatial distribution of these EQs with $\mathrm{M}_{L}>2.5$ (Fig. 1) completely overlaps with the area outlined by seismic activity over the past 45 years. The 2 largest EQs occurred; EQ1 on 17 April $2006\left(\mathrm{M}_{L}=4.7, h=3.9 \mathrm{~km}, 17.13 \mathrm{~N}\right.$, 73.78 E, 16.39.59.4 UT) and EQ2 on 21 May $2006\left(\mathrm{M}_{L}=4.2\right.$, $h=5.1 \mathrm{~km}, 17.17 \mathrm{~N}, 73.77 \mathrm{E}, 20.29 .01 .2 \mathrm{UT}$ ) and their fault plane solutions as determined by NGRI are shown as inset in Fig. 1. The ULF magnetic activity in relation to these 2 modest magnitude EQs is examined and presented in next sections.

\section{Experiment, results and discussion}

\subsection{Polarization analysis}

As described earlier, the polarization analysis in the form of $S_{Z} / S_{H}$ plots in the select frequency bands are commonly used to search precursory ULF magnetic signals before large earthquakes. Figure 2 gives the plot of $S_{Z} / S_{H}$ at a representative frequency band of $0.01-0.03 \mathrm{~Hz}$ (Yumoto et al., 2009) for the April-May 2006. This frequency band is selected as it is shown later that most precursory magnetic signals in ULF bands are concentrated in frequency range of $0.01-0.07 \mathrm{~Hz}$. This simple mode of presentation shows that except for oc-

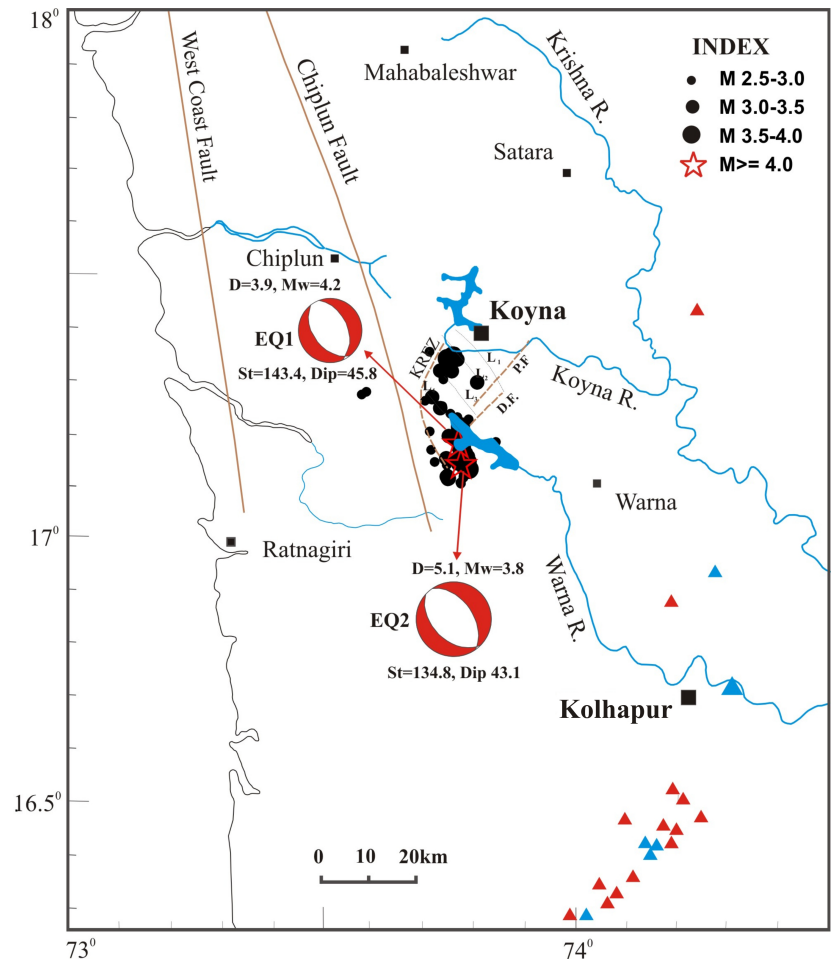

Fig. 1. Map of western India showing location of ULF magnetometer stations established to monitor EM emission related to reservoir triggered seismicity associated with Koyna-Warna reservoirs. Black dots represent epicentre of EQs recorded during the observational campaign period of March-June 2006. Fault plane solutions of two moderate EQs $\left(\mathrm{M}_{L}>4\right)$ are also shown. Surface intersection point of M-lines (see text) are indicated by red and blue triangles.

casional differences in the temporal variability, polarization ratios at both stations exhibit general resemblance. However, the variability of $S_{Z} / S_{H}$ ratios neither show any significant correlation with geomagnetic activity nor any significant change from background values, which could be classified as magnetic precursor candidates (see Fig. 2), is seen in relation to EQ occurrence. Given the high sensitivity of the LEMI-30 search coil magnetometer used here, magnetic signals with amplitude as low as $1-80 \mathrm{pT}$ on $H$ and $Z$ are well resolved but it seems likely that small amplitude EQ precursory magnetic signals, even if present in relation to the moderate magnitude EQ studied here, are completely masked by much stronger signals from ionospheric and magnetospheric sources to stand out clearly in $S_{Z} / S_{H}$ ratio plot.

\subsection{Source azimuth estimation by polarization ellipse method}

To extract information on the seismic source from the directional dependence of the ULF magnetic field components, the following data processing steps were carried out under some simplifying assumptions: 

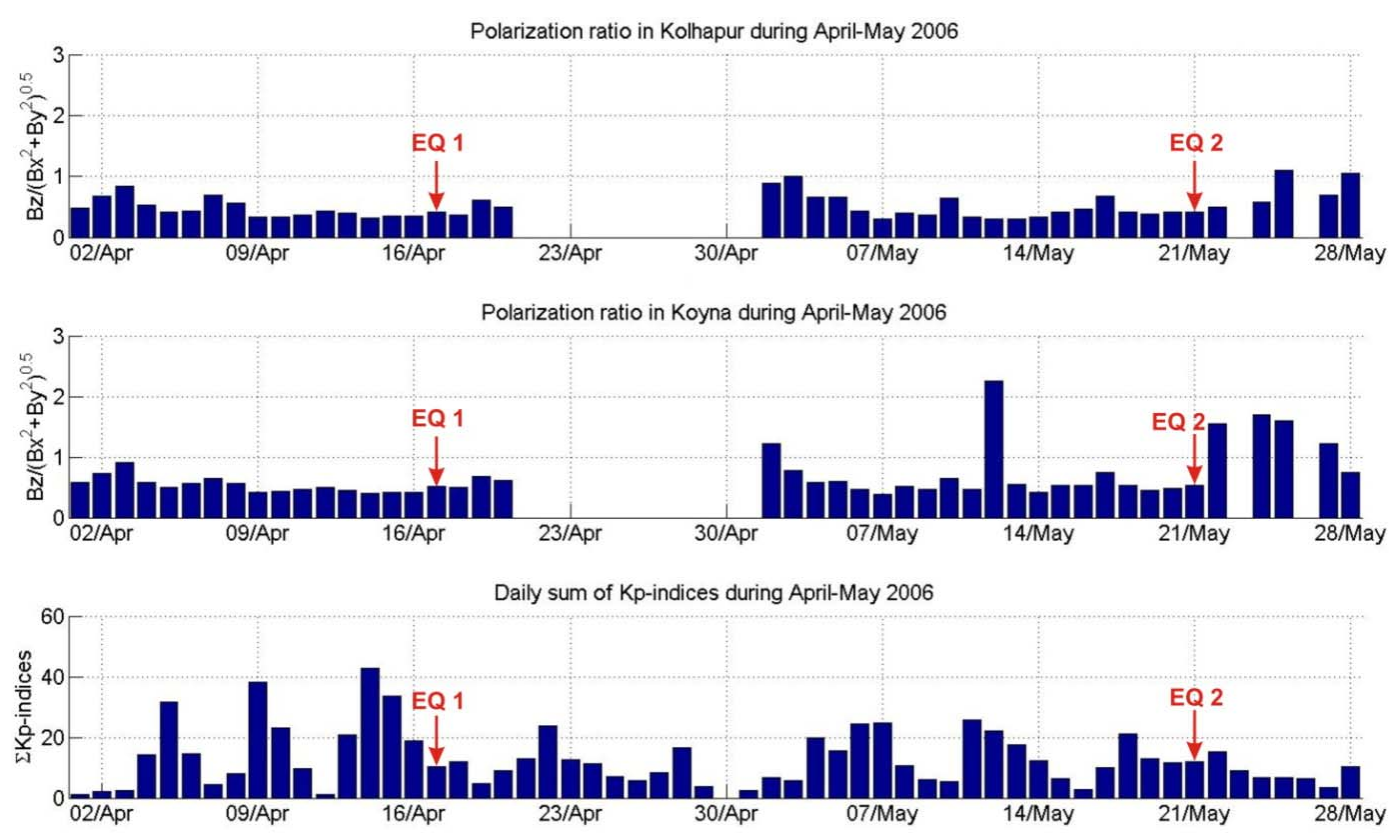

Fig. 2. Polarization ratio $S_{Z} / S_{H}$ in frequency range $0.01-0.03 \mathrm{~Hz}$ and $\mathrm{Kp}$ sums during March-June 2006. The gaps in data relate to days of mainpower disconnections.

1. In narrow ULF frequency band the magnetic field components can be represented as harmonic (periodic) signals.

2. At a given frequency, 3-orthogonal components of magnetic field with specific phases provide estimate the PE parameters, the resultant PE plane in space contains the source of EM field.

3. Alternating (induction) currents generated by any alternative seismo-EM source mechanisms, referred to in Sect. 1, can be visualized as small-scale superposition on large-scale telluric current system induced by global induction. These local current perturbations resulting from the motion of the conductive layer of rocks (inductive effects) or related to piezoelectric/piezomagnetic effects or so can generally be viewed as closed loop configuration and thus can be approximated to be equivalent to that produced by elementary magnetic dipoles placed in the source region. The assumption of magnetic-dipole type configuration is implicit in estimating the ULF source parameters using the spatial derivative of magnetic field (Surkov et al., 2004). In case of electrokinetic effect, the superposed current system may contain unclosed configuration (e.g. see Fedorov et al., 2001) and thus inhibit approximation of net flow by magnetic-type dipole. This would especially be valid when flow is confined to uniform homogeneous medium (Moore et al., 2004). However, for real crust characterized by inhomogeneous structures, it has been shown that when the underground water under the ef- fect of accumulating stresses is forced through the narrow fault plane the resulting streaming potential gives rise to concentrated flow of electric current along the fault plane that closes its path by way of return currents on either side of the fault plane (Mizutani and Ishido, 1976). This is consistent with the generalized calculations of Moore et al. (2004) who showed that net current resulting from electrokinetic effect consists of "impressed" and "back" (return) currents. In such a special structural geometry, the overall configuration of electrokinetic current flow can form compact asymmetric closed loops with a component of intense current along highly conductive duct (fault) and thus allowing magnetic dipole-like source approximation. Such approximation appears justified for the present study region where source region of seismicity is confined to short length $(\sim 20 \mathrm{~km})$ of narrow Koyna River Fault Zone (Gupta et al., 2007). This fault segment form steep boundary between inhomogeneous crustal blocks and provide conduits for fluid pressure flow which prevails right up to hypocenter depths (Talwani, 1997a; Pandey, 2003; Agrawal et al., 2004).

4. Magnetic moment of such a magnetic dipole source is in PE plane formed by its field components at the measurement points.

So far as the PE plane at any time contains the source of magnetic field (see Appendix), it is possible to find the intersection line of PE planes from observations at two distant stations (Fig. 3). This line, which we name as M-line, 


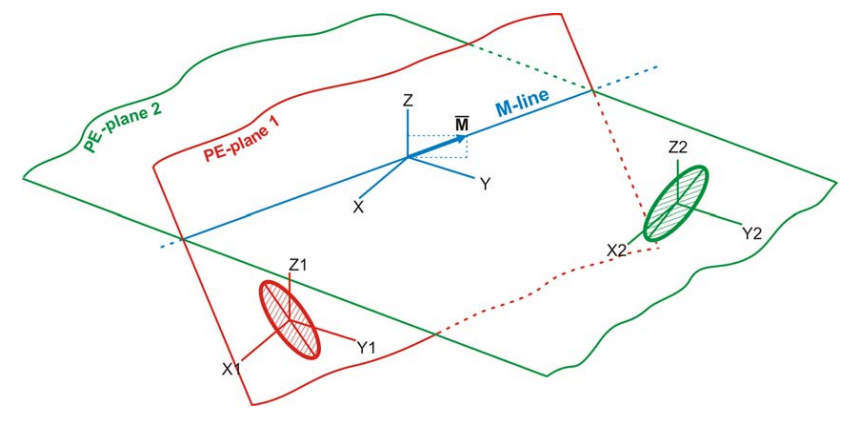

Fig. 3. Formation of M-line by the intersection of two PE-planes.

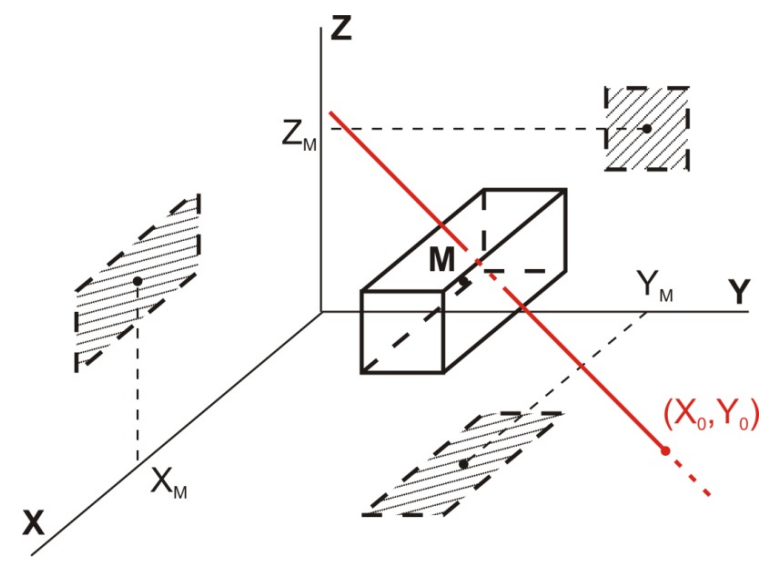

Fig. 4. Configuration of M-area.

contains the magnetic dipole moment $\boldsymbol{M}$, which is aligned along it, and can be calculated from parameters of PE. Calculation of PE parameters and magnetic moment follows the illustrations in Fig. A1a, b, the details can be found in relevant monographs on electromagnetism or in article (Morgan and Evans, 1951).

Source region of the EQs near Koyna is confined to narrow localized area $20 \times 30 \mathrm{~km}^{2}$ with hypocentres restricted to depths less than $12 \mathrm{~km}$ (Gupta, 2005; Gupta et al., 2007). Thus for the detection of the magnetic precursors of two main EQs, we defined the source area of interest (M-area) by a parallelepiped of $20 \mathrm{~km}$ length and a cross section of $3 \times 3 \mathrm{~km}^{2}$ with centre at EQ hypocentres. Then we calculate PE planes intersections (M-lines) which intersect M-area (Fig. 4) for both stations of synchronous observations. The M-lines were calculated for each point of DFS in the frequency range $0.001-0.5 \mathrm{~Hz}$ (i.e. for each harmonic and elementary time window used in the calculation of spectra).

It is widely accepted that the most promising frequency range of ULF magnetic precursors overlaps with the Pc3Pc5 band of micropulsations (see, for example, Hayakawa et al., 2007; Kopytenko et al., 2001; Molchanov et al., 2004). The sources of these pulsations are dominantly of magnetospheric origin related to field line resonance or large-scale

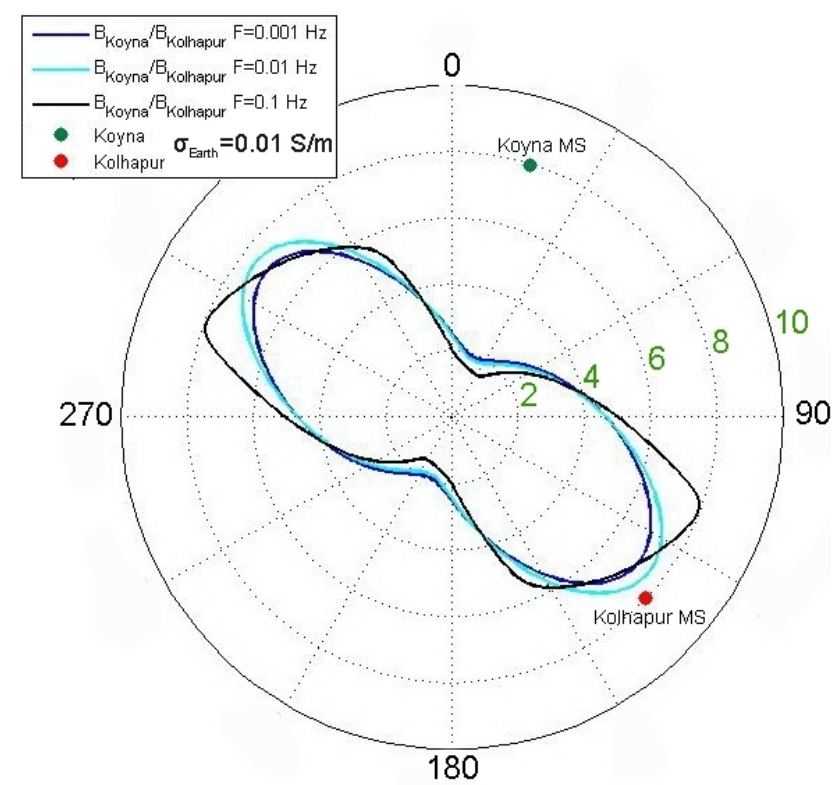

Fig. 5. Koyna/Kolhapur PE major axes ratio against direction of the magnetic dipole moment.

cavity oscillations (see for example Waters et al., 1994). The weak secondary ionospheric component arises due to instantaneous penetration of the magnetospheric inducted polar electric field to low and middle latitude (Trivedi et al., 1997). The spatial scale-length of these magnetospheric/ionospheric current system at low and middle latitudes are typically several thousand kilometers and, therefore, permits plane wave approximation for natural electromagnetic waves (Cagniard, 1953). A net consequence of the plane wave approximation on the present analysis is that the ratio of the amplitude of magnetospheric ULF magnetic signals at pair of measuring stations, separated by not more than couple of hundreds of $\mathrm{km}$, would be close to unity. In contrast, characteristics spatial scale-length of the ULF signal is no more than few hundred km (Hayakawa et al., 2007). Taking advantage of these large differences in dimension of the two sources, for the discrimination of M-lines associated with seismo-EM sources from natural background variations, we calculated the ratio of PE major axes in Koyna and Kolhapur measuring sites against orientation of horizontal magnetic dipole placed in EQ hypocentres by simplified formulas, see Appendix. The results of calculation are shown on Fig. 5, where the minimal ratio of PE major axes is about 2 . Therefore, it appears that allowing for contamination for background and man-made noise, the M-lines with PE major axes ratio exceeding the threshold value 2 can be ascribed to the ULF magnetic precursor candidate. 


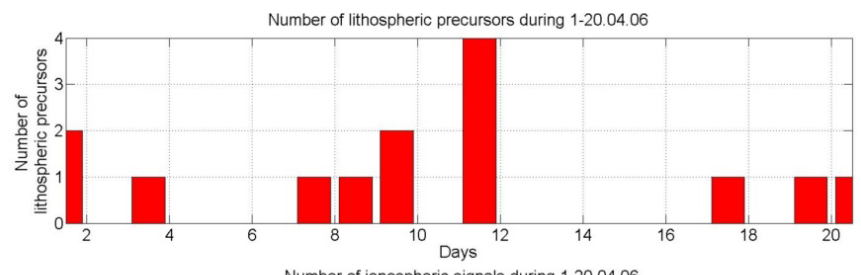

Number of ionospheric signals during 1-20.04.06

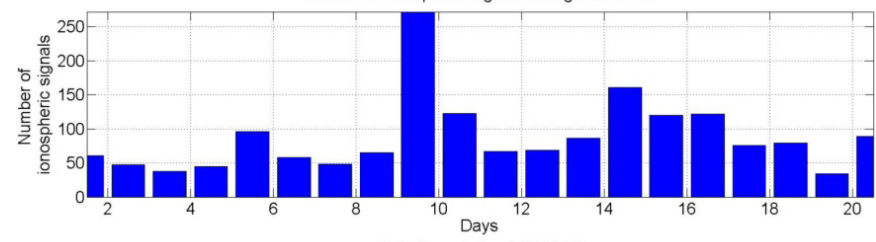

Kp-indices during 1-20.04.06

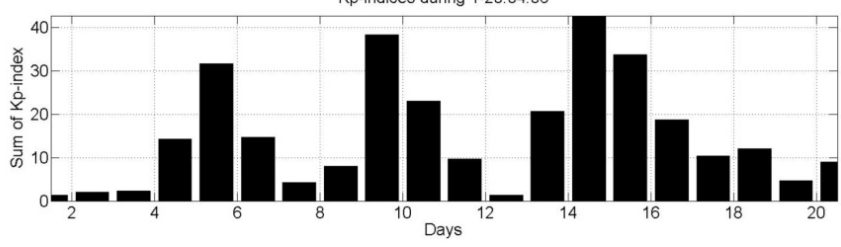

(a)

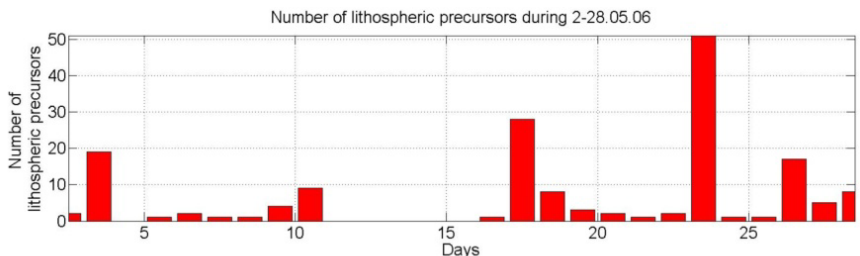

Number of ionospheric signals during 2-28.05.06

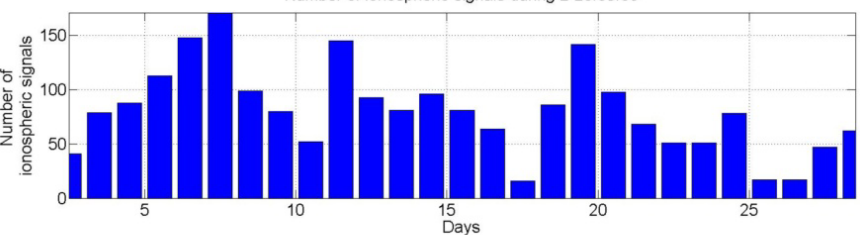

Days

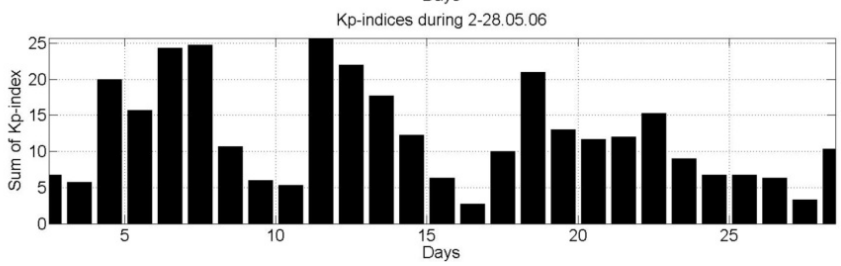

(b)

Fig. 6. The number of detected seismo-EM and ionospheric signals with the Kp-index values during time of observation. Red bars are seismo-EM precursors, blue bars are ionospheric signals.

\subsection{Source validation}

The detected and classified seismo-EM and ionospheric signals with the Kp-index values during the observation campaign interval are shown in Fig. 6a, b. The good correlation between the numbers of signals of the ionospheric origin and the value of Kp-index is clearly seen. The number of seismo-EM signals increase before EQ1 $\left(\mathrm{M}_{L}=4.7\right)$ up to 11 April and then approaches to zero level. After EQ1 very low seismo-EM activity in region of interest is observed. For EQ2 $\left(\mathrm{M}_{L}=4.2\right)$ the number of magnetic precursors is maximal on 17 May and then drop rapidly to small value. Then on 23 May the signals classified as seismo-EM origin rise again, which apparently is neither related to any abnormal seismic activity nor marked by intense solar/magnetic disturbances. However, given that this time interval is marked by moderate values of $\mathrm{Kp}$, it seems possible that these seismo-EM signals related to the release of residual mechanical stresses following EQ2.

Further as an example, the frequency of the seismo$\mathrm{EM}$ and ionospheric/magnetospheric signals against time are shown for 17, 18 and 20 May (see Fig. 7). The magnetic precursors are confined to narrow frequency range of about $0.01-0.07 \mathrm{~Hz}$ and completely overlap with the most dominant frequency range of ionospheric signals. Thus, source separation based on the frequency characteristic alone will not be unambiguous. However, it is shown that the ratio of major axes of PE at two distant sites proves effective in isolating EM signals of seismo-EM and in- ospheric/magnetospheric origins (Figs. 6 and 7). It may be added that fixing of major axes ratio at 2 for isolating signals of different origin is some what arbitrary. Adopting value below or above the critical threshold would, respectively, lead to either increased cases of contamination from ionospheric/magnetospheric sources or reduced number of precursory events for the purpose of further estimation of Mlines aimed at primary goal of source location. May be long term monitoring of magnetic fields in ULF band in a given seismic belt would help more realistic identification of factor.

\subsection{EM source mechanism and linkage to seismicity}

In the present study, M-lines estimations were done for all spectral lines by fixing major axes ratio at 2 . The cluster of points resulting from the intersection of calculated M-lines with the earth surface are superposed in Fig. 1 for all those spectral lines which are identified as possible magnetic precursor to the EQ2 (red dots in Fig. 7). It follows from the definition of M-lines, the line joining surface intersection points to the epicentral zone gives the azimuth of magnetic ULF source and similarly line connecting these surface interaction points with hypocentral zone gives the ascent angle of the source magnetic moment. It follows from Fig. 1 that azimuth of potential magnetic ULF precursors orient primarily in NNW-SSE direction. This direction closely follows the orientation of major lineament, mega fracture and geomorphic (fissure) features of the region (Talwani, 1997a). It is known that prolonged seismic activity in the Koyna-Warna 

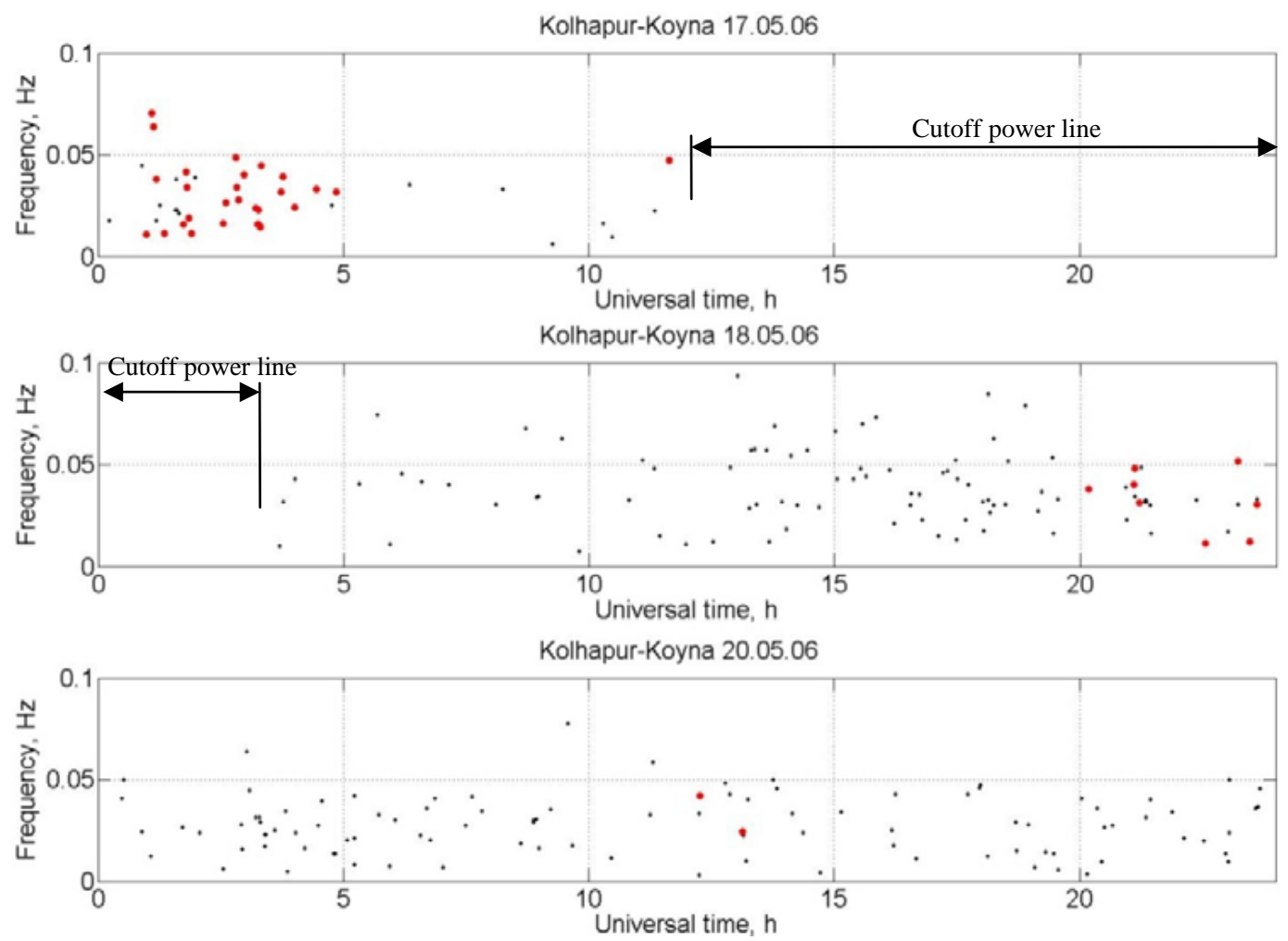

Fig. 7. Distribution of ionospheric and seismo-EM M-lines against time and frequency for 17, 18 and 20 May 2006. Red and blue points are seismo-EM and ionospheric signals respectively.

region starting from 1963 to the present has concentrated on a short $30 \mathrm{~km}$ long segment of the fault, which is seen as an extension of $10^{\circ} \mathrm{N}-15^{\circ} \mathrm{E}$ trending Koyna River Fault Zone (KRFZ) to the south in NNW-SSE direction (Fig. 1). Another notable feature of the seismicity in this region is that epicentres of all large EQs $(M>5)$ starting with the Koyna mainshock in $1967(M=6.3)$ to last EQ of $M>5$ in 2005 have progressively migrated southward on this NNW-SSE trending fault starting from the south of the Koyna river and extending to the Warna River (Talwani, 1997b). Amongst the several mechanisms suggested to explain the occurrence of continued seismicity in the Koyna-Warna region, the role of high-fluid pressure in producing failure on the preexisting critically stressed faults has received wider acceptance in generating EQs in the region (Talwani, 1997a; Pandey, 2003). More recently, extending this hypothesis, it have been attributed the temporal southward migratory trend in seismicity to similar changes in direction of pore-fluid pressure diffusion (Ramalingeswara and Singh, 2008). They further demonstrated that changes in fluid flow directions were independently corroborated by the strikes of the focal mechanisms of large EQs $(M>5)$. The fault plane solution of EQ1 and EQ2 indicate normal faulting, in agreement with the faults mechanisms for most of the large EQs recorded from this region (Gupta et al., 2002). Further, one of strike directions of fault plane solutions of the EQ1 and EQ2 is parallel to the active segment of the KRFZ. Given this correspondence and observational evidence that azimuth plane of magnetic ULF sources is oriented in the same direction, it is possible to postulate that both EQs and ULF magnetic signals are common manifestation of fluid flow diffusion in the focal zone. The active segment of the KRFZ, multiple fractures and fissure may be providing conduits for the high pore-pressure flow down to hypocentral depth (Talwani, 1997b; Agrawal et al., 2004). The oriented focused flow of fluid at focal depth under the effect of accumulated high stresses by way of electrokinetic effects may be the effective source cause for ULF magnetic signals (Mizutani et al., 1976; Uyeda et al., 2009, and references therein). The electrokinetic effect was earlier invoked by Arora (1988; also see Arora and Singh, 1992) to explain the bi-polarity nature of seismomagnetic anomalies observed around the Koyna reservoir in association with a sequence of moderate magnitude $\left(\mathrm{M}_{L}=4.1-4.3\right)$ EQs in the same area during 1980 .

\section{Summary}

The use of highly sensitive and low noise search coil LEMI30 magnetometers enabled to resolve ULF magnetic signals in frequency range of $0.001-0.5 \mathrm{~Hz}$ with amplitudes as low 
as of few pT. Despite this advantage, the commonly employed polarization analysis $\left(S_{Z} / S_{H}\right.$ ratio) fails to reveal any precursory seismo-EM signals, perhaps such signals, even if present, are completely masked by the much stronger signals of natural ionospheric/magnetospheric origin. This is rather not surprising as almost all reported anomalies are in relation to large EQs of $M>5$, whereas the only two EQs searched here have magnitude $\mathrm{M}_{L}>4$. However, controlled by the orientation of seismogenic faults, resulting seismo-EM field would have definite orientation in comparison to the isotropic direction distribution of highly variable natural signals arising from complex ionospheric-magnetospheric interactions. Based on these physical considerations, the interactions lines defined by the planes of PE, formed by the magnetic fields at minimum two sites, define the azimuth of seismo-EM source. Further, ratio of major axes of PEs above certain threshold help to distinguish ULF signals dominated by seismoEM origin from those associated with ionospheric origin. In the present case, this threshold is fixed at 2, corresponding to the minimum value of the ratio recorded between Koyna and Kolhapur. This choice of the value of cutoff threshold adopted here has arbitrariness but can be constrained better with long term data. Approximating the seismo-EM source as elementary magnetic dipole, the large numbers of spectral lines qualifying this threshold provide statistically averaged azimuth of the seismo-EM field. The NNW-SSE orientation of seismogenic ULF signals in the Koyna-Warna corresponds well with causative fault zone inferred from longterm EQ data. The already available knowledge on the role of high pressure fluids in generating the EQs favours electrokinetic effect to be one of the possible source mechanisms for seismo-EM fields. The alternative source mechanisms, inductive or piezomagnetic effects, may equally well explain the observations. Testing the proposed formulation to the other more active seismic belts, where source zone is not as well defined as the Koyna-Warna region would help generalization of the methodology for future EQ precursory studies. Such future experimentation essentially employing multiple stations would be key diagnostic to map the exact nature of sesimo-EM field perturbations and thus validating the magnetic dipole-like approximation for the source, implicit to the formulation advanced here.

\section{Appendix A}

The EM field of magnetic dipole with a moment $\mathrm{M}$ in spherical coordinate system has a following form (see Fig. A1a)

$$
\left.\begin{array}{l}
\boldsymbol{B}_{r}=\left(\boldsymbol{M} \mu \cos \theta / 2 \pi r^{3}\right)(1+j k r) \exp (-j k r), \\
\boldsymbol{B}_{\theta}=\left(\boldsymbol{M} \mu \sin \theta / 4 \pi r^{3}\right)\left(1+j k r-(k r)^{2}\right) \exp (-j k r), \\
E_{\varphi}=\left(\boldsymbol{M} \mu \sin \theta / 4 \pi r^{2}\right)(-j \omega)(1+j k r) \exp (-j k r),
\end{array}\right\},
$$

where $B$ is the magnetic induction; $E$ is the electric field strength; $\mu$ is the magnetic permeability of the medium; $k=$ $(\omega \mu \dot{\sigma})^{0.5}$ is the wave number of the medium, $\dot{\sigma}=\omega \varepsilon-j \sigma$;

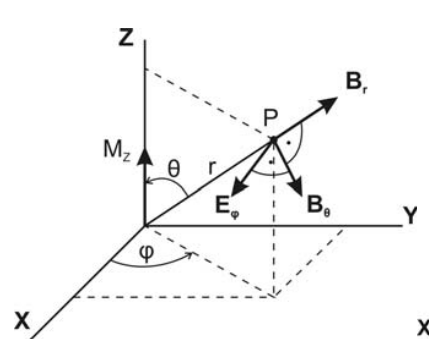

(a)

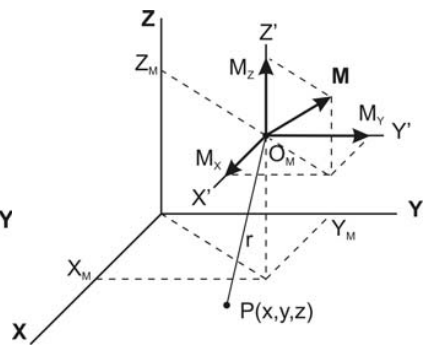

(b)
Fig. A1. Magnetic dipole source and components of EM field.

$\varepsilon, \sigma$ are the electric permeability and electric conductivity of the medium, respectively; $j=\sqrt{-1}, \omega=2 \pi f, f$ is the frequency of EM field, $\theta$ is angle between vectors of momentum $\boldsymbol{M}$ and $\boldsymbol{r}, \boldsymbol{r}$ is the distance between magnetic source and the point of observation. From Fig. A1a we can see that both components of magnetic field and consequently a PE are in the plane formed by vectors $\boldsymbol{M}$ and $\boldsymbol{r}$.

The magnetic field of arbitrary oriented magnetic dipole can be decomposed into horizontal and vertical dipoles (Fig. A1b). The directional diagram of such a dipole depends only on horizontal component of its magnetic moment.

The field of the horizontal magnetic dipole embedded in lossy medium (crust) for near field range approximation $\left(5 \rho_{A} \leq \rho \leq 0.2 \rho_{B}\right)$, where

$$
\begin{aligned}
\rho_{A} & =\left(10^{4} / 4 \pi\right)(0.2 / f \sigma)^{0.5}, \\
\rho_{B} & =3 \times 10^{8} /(2 \pi f), \\
\rho & =\left(x^{2}+y^{2}\right),
\end{aligned}
$$

( $\rho$ is the horizontal distance to dipole) for point of observation $(x, y, z)$ what is close to air-earth boundary $(z \ll h)$ can be written in the simplified form Baños (1966)

$$
\left.\begin{array}{l}
B_{\rho}=\left(\boldsymbol{M} \mu \cos \varphi / \pi \rho^{3}\right) \exp (j k(h-z)), \\
B_{\varphi}=\left(\boldsymbol{M} \mu \sin \varphi / 2 \pi \rho^{3}\right) \exp (j k(h-z)), \\
B_{z}=\left(3 j \boldsymbol{M} \mu \cos \varphi / 2 \pi k \rho^{4}\right) \exp (j k(h-z)),
\end{array}\right\}
$$

where $\rho, \varphi, z$ is the cylindrical coordinate system. The horizontal magnetic dipole has coordinates $(0,0,-h)$. For example, at the hypocenter depth $h=10 \mathrm{~km}$, frequency $0.5 \mathrm{~Hz}$ and the crustal conductivity $\sigma=0.01 \mathrm{~S} / \mathrm{m}, \rho_{A} \approx 5 \mathrm{~km}, \rho_{B} \approx$ $9.5 \times 10^{4} \mathrm{~km}$.

Acknowledgements. At Wadia Institute of Himalayan Geology, the work presented above was supported by the Ministry of Earth Sciences, Government of India under a sponsored project "Setting up of Multi-Parametric Geophysical Observatories for Earthquake Precursory Research", while part of the analysis at Lviv Centre was partially supported by the STCU grants 3165 . The magnetometer campaign, described here, was initiated on the advice of Harsh K. Gupta, Chairman of the Program Advisory Group. Many formal discussions with him about the Koyna seismicity 
and guidance are greatly acknowledged. B. K. Bansal, Program Co-ordinator, MoES is thanked for facilitating the activity and suggestions in experiment planning. Authors have special thanks to D. Shashidhar, NGRI, Hyderabad for readily providing the EQ data including the fault plane solutions. The help received from Shri D. D. Khandelwal in field campaign is deeply appreciated. Authors thank the parent institutions for providing facilities in bringing the present study to this stage.

Edited by: M. E. Contadakis

Reviewed by: A. De Santis and another anonymous referee

\section{References}

Agrawal, P. K., Pandey, O. P., and Chetty, T. R. K.: Aeromagnetic anomalies, lineaments and seismicity in Koyna-Warna Region, J. Ind. Geophys. Union, 8(4), 229-242, 2004.

Arora, B. R.: Tectonomagnetic studies in India (Invited), "Earthquake Prediction - Present Status", edited by: Guha, S. K. and Patwardhan, A. M., Dept. of Geology, University of Poona, Pune, India, pp. 53-62, 1988.

Arora, B. R. and Singh, B. P.: Geomagnetic and geoelectric investigations for seismicity and seismotectonics of the Himalayan region, in: Himalayan Seismicity, edited by: Gupta, G. D., Mem. Geol. Soc. India., 23, 223-262, 1992.

Baños, A.: Dipole radiation in the presence of a conducting halfspace, Pergamon Press, Oxford, 1966.

Bleier, T., Dunson, C., Maniscalco, M., Bryant, N., Bambery, R., and Freund, F.: Investigation of ULF magnetic pulsations, air conductivity changes, and infra red signatures associated with the 30 October Alum Rock M5.4 earthquake, Nat. Hazards Earth Syst. Sci., 9, 585-603, doi:10.5194/nhess-9-585-2009, 2009.

Cagniard, L.: Basic theory of the magnetotelluric method of geophysical prospecting, Geophysics, 18, 605-635, 1953.

Du, A., Huang, Q., and Yang, S.: Epicenter location by abnormal ULF electromagnetic emissions, Geophys. Res. Lett., 29(10), 1455-1458, 2002.

Dudkin, F., De Santis, A., and Korepanov, V.: Active EM sounding for early warning of earthquakes and volcanic eruptions, Phys. Earth Planet. Inter., 139(3, 4), 187-195, 2003.

Dudkin, F., Leontyeva, O., Arora, B. R., Rawat, G., and Sharma, A.: Analysis of magnetic field polarization parameters before and after Koyna earthquakes, EGU General Assembly, Vienna, Austria, 13-18 April 2008, Geophys. Res. Abstr., 10, EGU2008A-00054, 2008.

Fedorov, E., Pilipenko, V., and Uyeda, S.: Electric and Magnetic Fields Generated by Electrokinetic Processes in a Conductive Crust, Phys. Chem. Earth (C), 26(10-12), 793-799, 2001.

Fitterman, D. V.: Theory of electrokinetic magnetic anomalies in a faulted half-space, J. Geophys. Res., 84(B11), 6031-6040, 1979.

Fraser-Smith, A. C., Bernardi, A., McGill, P. R., Ladd, M. E., Helliwell, R. A., and Villard Jr., O. G.: Low-frequency magnetic field measurements near the epicenter of the Ms 7.1 Loma Prieta earthquake; Geophys. Res. Lett., 17, 1465-1468, 1990.

Gupta, H. K.: A review of recent studies of triggered earthquakes by artificial water reservoirs with special emphasis on earthquakes in Koyna, India, Earth-Sci. Rev., 58, 279-310, 2002.

Gupta, H. K.: Artificial water reservoir-triggered earthquakes with special emphasis at Koyna, Current Science, 88(1), 1628-1631,
2005.

Gupta, H., Shashidhar, D., Pereira, M., Mandal, P., Purnachandra, Rao N., Kousalya, M., Satyanarayana, H. V. S., and Dimri, V. P.: Earthquake forecast appears feasible at Koyna, India, Current Sci., 93(6), 1628-1631, 2007.

Hayakawa, M., Kawate, R., Molchanov, O. A., and Yumoto, K.: Results of ultra-low-frequency magnetic field measurements during the Guam earthquake of 8 August 1993, Geophys. Res. Lett., 23, 241-244, 1996.

Hayakawa, M., Itoh, T., and Smirnova, N.: Fractal analysis of ULF geomagnetic data associated with the Guam earthquake on $8 \mathrm{Au}-$ gust 1993, Geophys. Res. Lett., 26, 2797-2800, 1999.

Hayakawa, M., Itoh, T., Hattori, K., and Yumoto, K.: ULF electromagnetic precursors for an earthquake in Biak, Indonesia on 17 February 1966, Geophys. Res. Lett., 27, 1531-1534, 2000.

Hayakawa, M., Molchanov, O. A., and NASDA/UEC team: Achievements of NASDA's Earthquake Remote Sensing Frontier Project, TAO, 15(3), 311-327, 2004.

Hayakawa, M., Hattori, K., and Ohta, K.: Monitoring of ULF (ultralow-frequency) Geomagnetic Variations Associated with Earthquakes, Sensors, 7, 1108-1122, 2007.

Ida, Y. and Hayakawa, M.: Fractal analysis for the ULF data during the 1993 Guam earthquake to study prefracture criticality, Nonlin. Processes Geophys., 13, 409-412, doi:10.5194/npg-13409-2006, 2006.

Ismaguilov, V. S., Kopytenko, Yu. A., Hattori, K., and Hayakawa, M.: Variations of phase velocity and gradient values of ULF geomagnetic disturbances connected with the Izu strong earthquakes, Nat. Hazards Earth Syst. Sci., 3, 211-215, doi:10.5194/nhess-3-211-2003, 2003.

Johnston, M. J. S., Muller, J. S., and Sasai, Y.: Magnetic field observations in the near field: the 28 June, $1992 \mathrm{Mw} 7.3$ Landers, California Earthquake, B. Seism. Soc. Am., 84, 792-798, 1994.

Kopytenko, Yu. A., Ismaguilov, V. S., Hayakawa, M., Smirnova, N., Troyan, V., and Peterson, T.: Investigation of the ULF electromagnetic phenomena related to earthquakes: contemporary achievements and perspectives, Annali di Geofisica, 44(2), 325334, 2001.

Kopytenko, Yu. A., Ismaguilov, V. S., Hattory, K., and Hayakawa, M.: Determination of hearth position of forthcoming strong EQ using gradients and phase velocities of ULF geomagnetic disturbances, Phys. Chem. Earth, 31, 292-298, 2006.

Martin, R. J., Habermann, R. E., and Wyss, M.: The effect of stress cycling and inelastic volumetric strain on remanent magnetization, J. Geophys. Res., 83, 3485-3496, 1978.

Mizutani, H. and Ishido, T.: A new interpretation of the magnetic field variation associated with the Matsushiro Earthquakes, J. Geomagn. Geoelectr., 28, 179-188, 1976.

Mizutani, H., Ishido, T., Yokokura, T., and Ohnishi, S.: Electrokinetic phenomena associated with earthquakes, Geophys. Res. Lett., 13, 365-368, 1976.

Molchanov, O. A., Kopytenko, Yu. A., Voronov, P. M., Kopytenko, E. A., Matiashvili, T. G., Fraser-Smith, A. C., and Bernardy, A.: Results of ULF magnetic field measurements near the epicenters of the Spitak $(\mathrm{Ms}=6.9)$ and Loma Prieta $(\mathrm{Ms}=7.1)$ earthquakes: comparative analysis, Geophys. Res. Lett., 19, 1495-1498, 1992.

Molchanov, O. A. and Hayakawa, M.: Generation of ULF electromagnetic emissions by microfracturing, Geohpys. Res. Lett., 22, 3091-3094, 1995. 
Molchanov, O. A., Schekotov, A. Yu., Fedorov, E., Belyaev, G. G., Solovieva, M. S., and Hayakawa, M.: Preseismic ULF effect and possible interpretation, Ann. Geophys., 47(1), 119-131, 2004.

Moore, J. R., Glaser, S. D., Morrison, H. F., and Hoversten, G. M.: The streaming potential of liquid carbon dioxide in Berea sandstone, Geophys. Res. Lett., 31, L17610, doi:10.1029/2004GL020774, 2004.

Morgan, M. and Evans, W.: Synthesis and analysis of elliptic polarization loci in terms of space-quadrature sinusoidal components, Proc. IRE, 39, 552-556, 1951.

Ogawa, T., Oike, K., and Miura, T.: Electromagnetic Radiations from Rocks, J. Geophys. Res., 90(D4), 6245-6249, 1985.

Ogawa, T. and Utada, H.: Coseismic piezoelectric effects due to a dislocation. 1. An analytic far and early-time field solution in a homogeneous whole space, Phys. Earth Planet. Inter., 121, 273288, 2000.

Pandey, A. P. and Chadha, R. K.: Surface loading and triggered earthquakes in the Koyna-Warna region, western India, Phys. Earth Planet. Inter., 139, 207-223, 2003.

Ramalingeswara Rao, B. and Singh, C.: Temporal migration of earthquakes in Koyna-Warna (India) region by pore-fluid diffusion, J. Seismol., 12(4), 547-556, 2008.

Schekotov, A. Y., Molchanov, O. A., Hayakawa, M., Fedorov, E. N., Chebrov, V. N., Sinitsin, V. I., Gordeev, E. E., Belyaev, G. G., and Yagova, N. V.: ULF/ELF magnetic field variations from atmosphere induced by seismicity, Radio Sci., 42, RS6S90, doi:10.1029/2005RS003441, 2007.

Schekotov, A. Y., Molchanov, O. A., Hayakawa, M., Fedorov, E. N., Chebrov, V. N., Sinitsin, V. I., Gordeev, E. E., Andreevsky, S. E., Belyaev, G. G., Yagova, N. V., Gladishev, V. A., and Baransky, L. N.: About possibility to locate an EQ epicenter using parameters of ELF/ULF preseismic emission, Nat. Hazards Earth Syst. Sci., 8, 1237-1242, doi:10.5194/nhess-8-1237-2008, 2008.

Serita, A., Hattori, K., Yoshino, C., Hayakawa, M., and Isezaki, N.: Principal component analysis and singular spectrum analysis of ULF geomagnetic data associated with earthquakes, Nat. Hazards Earth Syst. Sci., 5, 685-689, doi:10.5194/nhess-5-6852005, 2005.
Surkov, V. V.: ULF electromagnetic perturbations resulting from the fracture and dilatancy in the earthquake preparation zone, pp. 357-370, in: Atmospheric and Ionospheric Phenomena Associated with Earthquakes, edited by: Hayakawa, M., TERRAPUB, Tokyo, 1999.

Surkov, V. V., Molchanov, O. A., and Hayakawa, M.: Preearthquake ULF electromagnetic perturbations as a result of inductive seismomagnetic phenomena during microfracturing, J. Atmos. Sol. Terr. Phys., 65(1), 31-46, 2003.

Surkov, V. V., Molchanov, O. A., and Hayakawa, M.: A direction finding technique for the ULF electromagnetic source, Nat. Hazards Earth Syst. Sci., 4, 513-517, doi:10.5194/nhess-4-5132004, 2004.

Talwani, P.: On the nature of reservoir-induced seismicity, Pure Appl. Geophys., 150, 473-492, 1997a.

Talwani, P.: Seismotectonics of the Koyna-Warna Area, India, Pure Appl. Geophys., 150, 511-550, 1997b.

Thomas, J. N., Love, J. J., and Johnston, M. J. S.: On the reported magnetic precursor of the 1989 Loma Prieta earthquake, Phys. Earth Planet. Int., 173, 207-215, 2009.

Trivedi, N. B., Arora, B. R., Padilha, A. L., Da Costa, J. M., and Dutra, S. L. G.: Global Pc5 geomagnetic pulsations of March 24, 1991, as observed along the American sector, Geophys. Res. Lett., 24, 1683-1686, 1997.

Uyeda, S., Nagao, T., and Kamogava, M.: Short-term earthquake prediction: Current status of seismo-electromagnetics, Tectonophysics, 470, 205-213, 2009.

Yumoto, K., Ikemoto, S., Cardinal, M. G., Hayakawa, M., Hattori, K., Liu, J. Y., Saroso, S., Ruhimat, M., Husni, M., Widarto, D., Ramos, E., McNamara, D., Otadoy, R. E., Yumul, G., Ebora, R., and Servando, N.: A new ULF wave analysis for SeismoElectromagnetics using CPMN/MAGDAS data, Phys. Chem. Earth, 34, 360-366, 2009.

Waters, C. L., Menk, F. W., and Fraser, B. J.: Low latitude geomagnetic field line resonance: Experiment and modeling, J. Geophys. Res., 99(A9), 17547-17558, 1994. 